\title{
Trust Evaluation for Data Exchange in Vehicular Networks
}

\author{
Nguyen Binh Truong \\ Liverpool John Moores University (LJMU) \\ Liverpool, United Kingdom, L33AF \\ n.b.truong@2015.ljmu.ac.uk
}

\author{
Gyu Myoung Lee \\ Liverpool John Moores University (LJMU) \\ Liverpool, United Kingdom, L33AF \\ g.m.lee@ljmu.ac.uk
}

\begin{abstract}
In Vehicular (Ad-hoc) Network (VANET), besides Vehicle-to-Vehicle communications (V2V), vehicles in VANET also exchange data with Road-Side-Units (RSUs) and Cellular Base Stations (Vehicleto-Infrastructure communications (V2I)). With the introduction of Intelligent Transport Systems (ITS), VANETs possess a great potential in enabling surveillance services. The rapid development of wireless communication technologies recently results in empowering data exchange among vehicles, RSUs and Cellular Base Stations, improving various types of applications and services such as safety driving, route planning, traffic alert, and context-aware infotainment. However, the benefits offered by VANETs and ITS cannot be fully realized unless there is a mechanism to effectively defend against fake and erroneous information exchange from malicious or dysfunctional nodes to other vehicles and RSUs for their own purposes. In this regards, trust appears as one of the solutions for VANETs to establish secure connectivity and reliable services. The conceptual idea to tackle down this challenges is that only data from trusted vehicles is taken into account. Thus, the aim is to evaluate trust of a vehicle in exchanging high quality of information. This paper presents the trust concept, key characteristics, a trust evaluation model, and a prototype for trusted data exchange activities in VANETs.
\end{abstract}

\section{KEYWORDS}

Vehicular Networks, VANET, Trust, Reputation, Experience, Knowledge, REK, Data Exchange

Nguyen Binh Truong and Gyu Myoung Lee. 2017. Trust Evaluation for Data Exchange in Vehicular Networks.

\section{INTRODUCTION}

Trust is a complicated concept used in many disciplines in human life, depending on both participators and environmental factors. It is widely acceptable that trust is as a fibelieffi of a trustor in a trustee to well (in some degree) perform a goal in a risky scenario [4]. Trust is a relationship between a trustor and a trustee; it is neither a property of the trustee nor the property of the trustor. A definition of trust is proposed as follows:

Trust is a belief of a trustor in a trustee that the trustee will provide or accomplish a trust goal as trustorfis expectation within a specific context for a specific period of time.

In this regard, the trustor partly recognizes the vulnerabilities and potential risks when the trustee accomplishes the goal, thus it represents the trustorfis willingness to be vulnerable under the conditions of risks and interdependence. Trust plays a crucial role in supporting humans, vehicles and services to overcome the perception of uncertainty and risk before making decision. The data exchange scenario in VANETs can be characterizing in detail as following:

- Task to accomplish: A vehicle exchanges data about an accident, traffic congestion, diversion, or infotainment to other vehicles, to a RSU or a Cellular Base Station.

- Requirements: (i) The task is event-based, spatial, and the data should reflect traffic situations on road correctly. (ii) Data transmission should be transmit in a secure manner, and it should not be compromised.

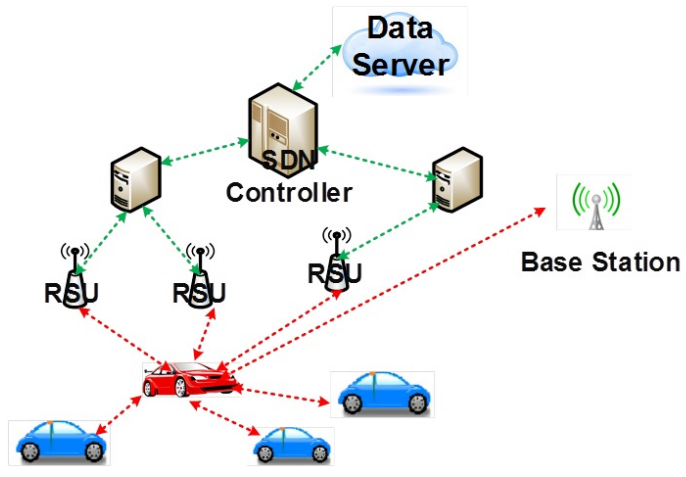

Figure 1: Data Exchange in Software Define Networkingbased VANET.

In this scenario, trustors can be vehicles, RSUs or Cellular Base Station whereas trustees are vehicles. Fig.1 illustrates the Data Exchange scenario in the a hybrid VANET architecture leveraging Software Defined Networking (SDN) with Fog Computing [6] in 
which the red car is the trustee exchanging data with other vehicles (blue cars), with RSUs and Cellular Base Station. Then, aim of evaluating trust for this use-case is clarified as following:

Evaluate trust between a vehicle, a RSU or a Base Station (trustors) to a vehicle (trustee) that the trustee will provide information (trust goal) that accurately describes what happens on road (high quality information as expectation).

\section{REK TRUST EVALUATION MODEL}

Psychologically, human usually bases on three indicators (TIs) for trust: public evidences on trustee (as Reputation), own experiences on the trustee (as Experience), and his understandings about trustee (as Knowledge). Some researchers have considered reputation (and recommendation) as indirect trust (or third-party information); and knowledge as direct trust (or direct observation). We believe this social phenomenon could be applied as the so-called REK trust evaluation model in the VANETs environment (Fig. 2).

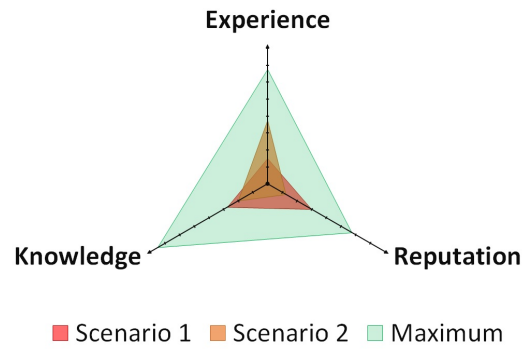

Figure 2: REK Trust Evaluation Model comprised of the triad Reputation fi Experience fi Knowledge indicators.

- Reputation TI: Reflects global opinion on the trustee by aggregating experiences of other entities on the trustee. It indicates how well the trustee has exchanged data with all of the entities until now. The Reputation TI calculation can be done in a centralized system where all of the previous interactions and Experiences TI are kept track of. The Software Define Networking-based VANET architecture is suitable because the evaluation mechanism can be deployed at the SDN Controller (Fig. 1).

- Experience TI: Reflects personal observation on the trustee by accumulating previous interactions of the trustor with the trustee. It indicates that how well the trustor have accomplished exchanging data with the trustee until now. The Experience TI calculation mechanism can be deploy in both distributed and centralized manners. Each of entities (vehicles, RSU, Base Station) either can keep track of its interactions with others by itself (local storage and computation) or Experience TI can be done in a centralized system like the SDN Controller.

- Knowledge TI: Renders observation on the trustee (the vehicle which is providing data) as direct trust. Some trust attributes are necessary to consider when calculating Knowledge such as communication capability to transmit data (including WiFi, WiMax, 3G, LTE, Signal Strength, and Bit-Error-Rate ), secure data transmit channel (data encryption, and authentication mechanisms), honesty (indicating of keeping promise in the social networks), cooperativeness (indicating how cooperativeness of trustee when interacting with others), credibility and correctness (indicating the quality of previous data provided by the users).

Base on the values of the triad Reputation, Experience, and Knowledge TIs, an overall trust value is aggregated as the outcome of the evaluation model. Technically, there are two canonical methods to derive TIs from associated attributes; and derive the overall trust value from the three TIs. The choice which method is appropriate for the calculation depends on specific situations. The first approach is to use mathematical models such as weighted sum, Bayesian neutron networks [1][3], reputation heuristic algorithms [5] or Google PageRank algorithm [2]. Normally, this approach is used to measure some quantitative attributes as well as Experience and Reputation TIs. The second method makes use of an inference engine for inferring new trust-related knowledge from an already known knowledge base. This reasoning mechanism is frequently used for deriving some causal-consequence and subjective attributes and also for deriving the overall trust value.

\section{CONCLUSIONS AND FUTURE WORK}

We have clarified the concept of trust in data exchange in VANETs. A trust evaluation model called REK incorporating both direct trust and indirect trust in terms of Knowledge, Reputation, and Experience TIs has proposed. A brief description and tentative mechanisms for calculating the three TIs is also presented. This work opens numbers of research directions to fulfil the development of the trust platform. The first is detail mathematical models and mechanisms for the TIs. Another direction is a simulation for data exchange in VANET in which trust can be actually measured. The third could be an autonomous mechanism to evaluate trust in a dynamic environment like VANETs in which trust-related data frequently updates and changes, resulting in changes of the trustrelated evaluation values

\section{ACKNOWLEDGMENTS}

This research was supported by the ICT R\&D program of MSIP/IITP [R0190-15-2027, Development of TII (Trusted Information Infrastructure) S/W Framework for Realizing Trustworthy IoT Eco-system] and EU funded Horizon 2020 Wise-IoT project [Worldwide Interoperability for Semantics IoT].

\section{REFERENCES}

[1] Fenye Bao, Ray Chen, and Jia Guo. 2013. Scalable, adaptive and survivable trust management for community of interest based internet of things systems. In Autonomous Decentralized Systems (ISADS), 2013 IEEE Eleventh International Symposium on. IEEE, 1-7.

[2] Sergey Brin and Lawrence Page. 2012. Reprint of: The anatomy of a large-scale hypertextual web search engine. Computer Networks 56, 18 (2012), 3825-3833.

[3] Ray Chen and Jia Guo. 2014. Dynamic hierarchical trust management of mobile groups and its application to misbehaving node detection. In Advanced Information Networking and Applications (AINA), 2014 IEEE 28th International Conference on. IEEE, 49-56.

[4] Francisco J Martinez, Chai-Keong Toh, Juan-Carlos Cano, Carlos T Calafate, and Pietro Manzoni. 2010. Emergency services in future intelligent transportation systems based on vehicular communication networks. IEEE Intelligent Transportation Systems Magazine 2, 2 (2010), 6-20.

[5] Paul Resnick, Ko Kuwabara, Richard Zeckhauser, and Eric Friedman. 2000. Reputation systems. Commun. ACM 43, 12 (2000), 45-48.

[6] Nguyen B Truong, Gyu Myoung Lee, and Yacine Ghamri-Doudane. 2015. Software defined networking-based vehicular adhoc network with fog computing. In Integrated Network Management (IM), 2015 IFIP/IEEE International Symposium on. IEEE, 1202-1207. 